魏明灯*，魏巧华

福州大学化学学院, 福建省新型电化学储能材料重点实验室, 福州 350108

摘要: 以 “碱金属的结构与性能及其在锂离子电池中的应用” 为例说明元素化学课堂教学中的方法, 旨在引导学生 学习与掌握碱金属的结构与性质的基础知识, 使学生对碱金属元素在锂离子电池中应用产生兴趣, 领悟无机化学相 关的科学前沿的新知识、新规律和新概念, 培养学生的创新意识。同时, 分组讨论碱金属的结构与化学性质之间关系 及其在新能源领域中的应用, 组织学生深入到开展相关研究的课题组, 在教师指导下开展锂离子电池电极材料制备 与表征以及器件组装和电化学性能测试, 并根据获得的结果复习课堂中讲授的知识。进一步, 鼓励和推进学生开展 碱金属在新能源器件中应用的本科生科研训练, 在科学研究中应用无机化学基础知识, 实现课本知识与科学实践相 衔接。

关键词: 元素化学; 课堂教学; 结构; 性能; 锂离子电池; 教学改革

中图分类号: G64; O6

\title{
Design of Classroom Teaching in Inorganic Chemistry: Structure and Properties of Alkali Metal Elements and Their Applications in Lithium- Ion Batteries
}

\author{
Mingdeng Wei ${ }^{*}$, Qiaohua Wei \\ Fujian Key Laboratory of Electrochemical Energy Storage Materials, College of Chemistry, Fuzhou University, \\ Fuzhou 350108, China.
}

\begin{abstract}
This paper uses "the structure and properties of alkali metal elements as well as their applications in lithium-ion battery" to illustrate the concrete methods in the classroom teaching of elementary chemistry. It is aimed at guiding the students to understand the structure and properties of alkali metal elements and arising their interest in applications of alkali metal elements in lithium-ion battery in the classroom land discussion. The relationships among the structure, chemical performance and applications in lithium-ion battery for alkali metal elements have been discussed in detail. Furthermore, a series of experiments including synthesis and characterization of electrode materials, and fabrication and measurement of devices, will be executed. Finally, the students have also been encouraged to execute "Students Research Training Program, SRTP" in new energy materials related institutes, and in this way the classroom knowledge and scientific research have been linked closely.
\end{abstract}

Key Words: Chemistry of elements; Classroom teaching; Structure; Performance; Lithium-ion battery; Teaching reform

收稿: 2020-08-28; 录用: 2020-09-23; 网络发表: 2020-11-26 
无机化学是学生进入大学后的第一门专业课, 需要面临教与学方式的根本转变。由于学生在中 学阶段大都已经接触到课程的知识点, 对教学内容认识不足, 产生轻视思想, 而社会对化学基础科 学的重要性缺乏正确认识, 尤其是與论对化学毕业生就业前景的误导, 致使不少学生刚入学就想着 转专业, 难以专心于课堂听讲。如何满足全体学生的知识需求和激发他们的听课兴趣, 成为课堂教 学的关键。实际上, 无机化学课程中的大多数知识点, 在下一学年的物理化学、结构化学等课程中 又会在更深的层次上重复出现。因此, 设计好课堂教学内容, 避免不必要的重复, 是十分重要的。同 时, 元素化学的课堂授课内容、授课效果将直接影响学生的未来出路。尤其是, 我校的化学学科正 在实施 “一流学科” 建设, 旨在培养出基础知识扎实、创新思维和实践能力突出的高素质基础学科 人才, 而无机化学课程的教学质量不仅影响学科建设的成效, 而且影响学生学习兴趣和创新意识, 还 会左右学生报考研究生专业方向。

本文以 “碱金属的结构与性能及其在锂离子电池中的应用” 为例, 介绍笔者面向化学专业理科 基地班一年级学生开展元素化学课堂所运用到的教学原则、教学内容、教学方法以及教学效果。

\section{1 授课对象及课程概况}

化学专业理科基地班的人才培养目标是培养出基础理论扎实、知识结构合理、富有创新意识和 实践能力, 能将化学基础理论知识与科研实践和生产实际相结合, 能进行化学基础科学研究的创新 型人才[1]。

作为化学专业理科基地班核心课之一的无机化学, 开设在一年级上下两个学期。无机化学I (48 学时), 主要讲授原子结构、化学键与分子结构、化学热力学、化学反应速率、化学平衡、溶液和氧 化还原反应等; 无机化学II (64学时), 主要讲授配位化学和元素化学, 其中元素化学授课学时为32学 时。实际上, 化学基础课程教学内容的设计对 “一流学科” 建设中化学专业理科基地班学生的创新 意识培养方面具有举足轻重的作用。因此, 元素化学课堂教学内容应该遵循以下原则 ${ }^{[2]}$ : 一是 “结构 决定性质”, 利用分子结构解释单质和化合物的物理与化学性质; 二是 “性质决定应用”, 讲授与元 素化学相关最新基础科学研究和应用方面的进展, 吸引学生的课堂注意力; 三是 “课堂知识与实践 结合”, 听课讨论和安排学生进入实验室, 开展电极材料制备和电池组装, 培养科研兴趣和创新意识。 同时, 采用课堂讲授与科研实践相结合方式, 使学生对元素性质及其应用有着更深层的认识, 也体 现出元素化学对社会和经济发展的重要性。以下具体说明 “碱金属的结构与性能及其在锂离子电池 中的应用” (2学时)的教学内容组织与实施方式。

\section{2 课堂教学的内容组织与实施方式}

\section{1 课堂教学引导}

教学过程从我们每个人日常生活中高度依赖的手机开始, 引导学生对手机不断轻量化过程的认 识, 并指出手机轻量化的最大贡献是手机电源—锂离子电池的更新换代。同时, 展示实验室锂离 子电池样品和播放彩色动画课件展示其工作原理, 引导学生认识锂离子在能量存储过程中扮演的角 色和电化学反应过程, 并详细地说明锂离子电池的构成成分及其作用。进一步, 结合中学化学课程 中所涉及的原电池方面的知识, 介绍电化学发展历史 ${ }^{[3]}$ 以及未来锂离子电池的发展方向 ${ }^{[4]}$ 和我国新 能源发展战略, 从而切入本课程的教学内容。

\subsection{1 碱金属元素的结构与性能}

碱金属在元素周期表中位于IA, 包括锂、钠、钾、铭、铯、钫六种金属元素, 其最外层电子排 布为 $n s^{1}$, 与碱土金属元素构成周期表 $s$ 区。碱金属元素的原子很容易失去一个电子而呈 +1 氧化态, 具有 很强的活泼性, 在自然界中难以以单质的形式存在。同时, 碱金属元素具有很大的第二电离能, 不 会表现出其他氧化态。实际上, 碱金属的性质都是由其结构决定的。

碱金属原子只有一个价电子且原子半径较大, 金属键弱, 导致其熔沸点低。碱金属是活泼的金 
属元素, 能够直接或者间接与电负性较高的非金属元素如卤素、硫、氧、磷、氮和氢等形成相应的 化合物。其中, 除了锂的某些化合物具有较为明显的共价性质外, 其他的碱金属化合物则具有离子 键的性质。碱金属的化学活泼程度还可以从它们与 $\mathrm{H}_{2} \mathrm{O}$ 作用中可以看出, 其中钠与 $\mathrm{H}_{2} \mathrm{O}$ 反应猛烈, $\mathrm{K}$ 、 $\mathrm{Rb}$ 和 $\mathrm{Cs}$ 遇 $\mathrm{H}_{2} \mathrm{O}$ 就发生燃烧, $\mathrm{Li}$ 与 $\mathrm{H}_{2} \mathrm{O}$ 反应则比较缓慢。实际上, 这些性质对其实际应用有着重大的影 响, 也就是 “性质决定应用”。

\subsection{2 碱金属氧化物和氢氧化物的结构和化学性质}

碱金属与氧所形成的氧化物, 包括氧化物 $\mathrm{M}_{2} \mathrm{O}$ 、过氧化物 $\mathrm{M}_{2} \mathrm{O}_{2}$ 、超氧化物 $\mathrm{MO}_{2}$ 和臭氧化物。氧 化物的热稳定性从 $\mathrm{Li}$ 到 $\mathrm{Cs}$ 逐渐降低, 除 $\mathrm{Li}_{2} \mathrm{O}$ 外, 其余碱金属氧化物需要在缺氧的环境中严格控制条 件才能获得。碱金属都能够生成含 $[\mathrm{O}-\mathrm{O}]^{2-}$ 离子的过氧化物, 可被看成过氧化氢 $(\mathrm{H}-\mathrm{O}-\mathrm{O}-\mathrm{H})$ 的盐, 但纯净碱金属过氧化物一般很难通过直接氧化碱金属而得到。 $\mathrm{K} 、 \mathrm{Rb} 、 \mathrm{Cs}$ 在过量的氧气中燃烧均可 产生超氧化物, 而制备 $\mathrm{NaO}_{2}$ 须在 $450^{\circ} \mathrm{C}$ 温度和 $15 \mathrm{MPa}$ 应力下通过 $\mathrm{Na}$ 与氧气反应获得。 $\mathrm{Na} 、 \mathrm{~K} 、 \mathrm{Rb}$ 、 $\mathrm{Cs}$ 的臭氧化物可以利用它们相应的固态氢氧化物与臭氧反应获得, 但是与 $\mathrm{H}_{2} \mathrm{O}$ 剧烈反应, 生产氢氧 化物, 如式(1)所示:

$$
4 \mathrm{MO}_{3}^{-}(\mathrm{s})+\mathrm{H}_{2} \mathrm{O}=4 \mathrm{MOH}+5 \mathrm{O}_{2}
$$

碱金属的氢氧化物是易溶于水的白色固态物, 其碱性强弱可以由金属阳离子的离子势 $\phi$ 值的大 小来确定, $\phi$ 为离子的电荷数 $Z$ 与离子半径 $r$ 之比, 如式(2)所示:

$$
\phi=Z / r
$$

由于碱金属氢氧化物的 $\phi$ 值均小于 0.22 , 发生碱式解离 $\left(\mathrm{M}-\mathrm{O}-\mathrm{H} \rightarrow \mathrm{M}^{+}+\mathrm{OH}^{-}\right)$, 容易呈碱性 ${ }^{[5]}$, 这也是其结构决定性质。

\section{2 组织学生讨论碱金属及其应用}

\subsection{1 锂离子电池的基础知识}

随着新能源汽车普及和风光储能电站建设, 作为储能器件的锂离子电池在我国能源体系中发 挥着越来越重要的作用。授课教师利用课件介绍锂离子电池的工作原理、发展过程以及2019年三位 诺贝尔化学奖获得者对锂电池的贡献。尤其是, 结合中学化学课本涉及到的原电池等方面的知识, 讲解锂离子电池的工作原理和电化学反应过程(如图1和式(3)-式(5)所示)以及它们之间的差异。

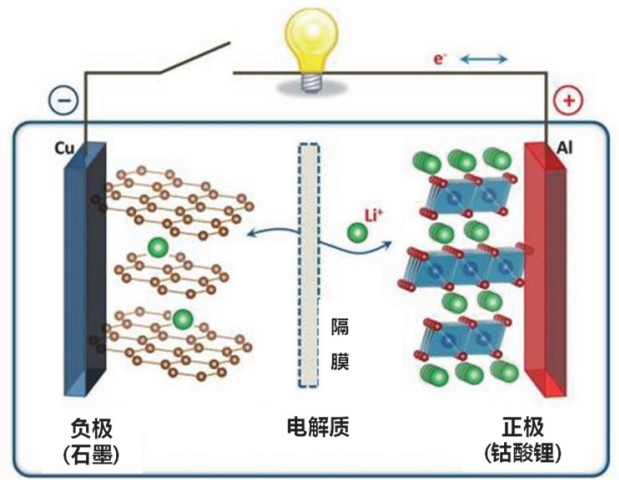

图1＼cjkstart锂离子电池工作原理示意图 ${ }^{[4]}$

正极反应: $\mathrm{LiCoO}_{2} \Leftrightarrow \mathrm{Li}_{1-x} \mathrm{CoO}_{2}+x \mathrm{Li}^{+}+x \mathrm{e}^{-}$

负极反应: $6 \mathrm{C}+x \mathrm{Li}^{+}+x \mathrm{e}^{-} \Leftrightarrow \mathrm{Li}_{x} \mathrm{C}_{6}$

总反应式: $6 \mathrm{C}+\mathrm{LiCoO}_{2} \Leftrightarrow \mathrm{Li}_{1-x} \mathrm{CoO}_{2}+\mathrm{Li}_{x} \mathrm{C}_{6}$

充电时, 受到外电场的驱动, 电池内部形成 $\mathrm{Li}^{+}$的浓度梯度, $\mathrm{Li}^{+}$从 $\mathrm{LiCoO}_{2}$ 的晶格中脱出, 进入电解 液后扩散到负极, 到达负极的 $\mathrm{Li}^{+}$嵌入到石墨的层间中, 嵌入的 $\mathrm{Li}^{+}$越多, 充电容量就越高, 而电子沿着 外电路从正极侧向负极移动。放电时, 嵌在石墨层间的 $\mathrm{Li}^{+}$脱出, 进入电解液后扩散到 $\mathrm{LiCoO}_{2}$ 正极并嵌 入晶格中, 电子沿着外电路从负极侧向正极移动。由于 $\mathrm{Li}^{+}$在正负极之间来回运动, 锂离子电池又被称 
为 “摇椅电池”。在锂离子电池中, 正极和负极材料是关键组成成分, 它们的比容量决定了器件的能量 密度和功率密度, 而电极材料的理论比容量可以根据式(6)计算 ${ }^{[6]}$ :

理论比容量 $=n F / 3.6 M$

式(6)中, $n$ 为每摩尔电极材料在氧化或还原反应中转移电子的量, $F$ 为法拉第常数 $(F=96485$ $\left.\mathrm{C} \cdot \mathrm{mol}^{-1}\right), n F$ 为转移总电荷量, $M$ 是电极材料的摩尔质量 $\left(\mathrm{g} \cdot \mathrm{mol}^{-1}\right)$ 。

很显然, 锂离子电池的电极材料选择是非常重要的, 因其本身固有特性决定了器件的性能, 也 就是 “结构决定性质”。另一方面, 碱金属元素也是锂离子电池中电解液的重要组成成分如六氟化磷 锂、四氟嗍酸锂、高氯酸锂等。电解液被称为锂电池的 “血液”, 在正负极之间起到传导电子的作用, 其性质显著地影响器件的能量密度和实际应用。

2018年我国锂离子电池的全球市场份额就突破了 $60 \%$, 占据世界第一。随着我国新能源汽车销 量增长和储能电站建设的扩大, 对锂离子电池的需求保持旺盛势头, 导致锂价格的飞涨。由于我国 锂资源短缺, 寻找替代碱金属锂是十分紧迫的。近年来, 钠离子和钾离子电池的研究进展十分迅速, 也取得了一系列成果 ${ }^{[7,8]}$ 。虽然它们的工作原理与锂离子电池相似, 但是, 较大离子半径的钠/钾离子 在电极材料中脱嵌困难, 而原子质量大也导致理论容量低, 从而影响器件的能量密度, 这实际上反 映出 “性质决定应用”。在此, 安排学生查阅相关文献, 了解国内外有关锂离子电池研究与产业的最 新进展, 为下一步进入实验室实习作必要的准备。

\subsection{2 锂离子电池的实验与讨论}

在课堂介绍锂离子电池基础知识的基础上, 通过演示和拆解锂电池样品, 让学生近距离地了解 元素化学与锂离子电池组成及其性能的关系, 并引导学生围绕着元素化学 “结构决定性质” 和 “性 质决定应用” 而展开师生之间和同学之间的讨论, 加深对元素性质的理解。在此, 教师可以顺势引 导学生关注锂离子电池领域的科技前沿问题。特别需要强调的是, 尽管我国在锂离子电池产业实现 了从跟踪到引领, 但是并没有拥有锂电池关键材料的核心专利和原创技术。在此, 教师借此机会介 绍本学院在锂离子电池领域的研究进展以及国内大型锂电池企业及其产业链的发展状况 ${ }^{[9,10]}$ 。同时, 安排学生课余时间进入实验室, 开展电极材料的制备、电池组装和电化学性能测试, 充分理解元素 化学的重要性, 实现课堂知识与实际应用的衔接。最后, 教师应该指出锂离子电池的能量密度是目 前制约新能源汽车发展的瓶颈和研制新型高性能电极材料的重要性, 激发学生报考研究生和从事新 能源相关领域研究的热情, 期望他们掌握元素化学的基础知识, 摆脱无机化学教材中的理论束缚, 潜心于探索和挑战, 获得颠覆性的重大原创性成果。

\subsection{3 教学效果}

据统计, 第一学年内超过一半学生利用周末时间分批进入实验室, 并在研究生和教师的指导下 开展电极材料制备、电池组装和性能测试, 详细了解锂电池的研制过程。同时, 他们带着课堂上学 到的元素化学基础知识, 与研究生和教师讨论元素性质与其电化学性能的关系。第二学年2组6个听 课学生申请了SRTP本科生科研训练项目, 第三学年又有 1 组 3 名学生申请, 他们怀着对科研的兴趣和 对化学的热爱, 利用课余时间开展为期一年有关锂电池材料与器件方面的研究, 并完成结题。第四 学年5名学生进入实验室完成有关锂电池研究方面的毕业实习和毕业论文, 并参与学术论文发表。另 一方面, 笔者多年来一直在讲授元素化学时融入电化学知识, 也间接地激发学生报考电化学专业研 究生的热情, 报考厦门大学能源化学专业和本校新能源材料专业的人数均超过 10 名, 并且部分毕业 生成功地应聘国内大型锂电池企业如宁德时代、厦门铇业等公司的相关岗位, 使他们有机会利用所 掌握的元素化学基础知识在学习和工作中发挥作用。

\section{3 结语}

元素化学作为无机化学的一个重要组成部分, 在无机化学课程教学中必须加以重视。在课堂教 学过程中, 首先从中学教材中已学过的原子结构、分子结构、原电池等入手, 以便学生容易理解结 
构与性质之间的构效关系; 其次, 授课教师介绍相关元素的发展历程和科学研究最新进展, 让学生 能够深刻理解和掌握元素和化合物性质及其应用; 最后, 教师应因势利导, 从国家发展战略高度说 明我国锂电池领域发展现状和面临的挑战, 并强调重大原创性成果的重要性, 引导学生关注国家和 地方经济发展的战略需求, 激发他们探索科学的热情, 启发他们的创新意识和投身化学领域研究的 决心。这样将有助于发挥化学基础课程无机化学教学对化学类创新人才培养的作用, 实现化学 “一 流学科”建设目标，提高我国基础科学领域的原始创新能力。

[1] 杨屹, 陈咏梅, 白守礼, 许家喜, 李蕾, 李保山. 中国大学教学, 2013, No. 7, 15.

[2] 陈咏梅, 周云山, 王桂荣, 金傘. 大学化学, 2019, 34 (5), 15.

[3] 吴宇平, 戴晓兵, 马军旗, 程预江. 锂离子电池一一应用于实践. 北京: 化学工业出版社, 2004: 1-12.

[4] Pistoia, G. 锂离子电池技术一一研究进展与应用. 赵瑞瑞, 余乐, 陈红雨, 译. 北京: 化学工业出版社, 2017: 4-12.

[5] 曹锡章, 宋天乔, 王杏乔. 无机化学. 北京: 高等教育出版社, 1978: 804-805.

[6] 彭佳悦, 祖晨㬢, 李泓. 储能科学与技术, 2013, 2 (1), 55 .

[7] Liang, Y. R.; Lai, W. H.; Miao, Z. C.; Chou, S. L. Small 2018, 14, 1702514.

[8] Zhang, W. C.; Liu, Y. J.; Guo, Z. P. Sci. Adv. 2019, 5, 7412.

[9] Zhang, W. F.; Zhang, Y.; Yu, L.; Wu, N. L.; Huang, H. T.; Wei, M. D. J. Mater. Chem. A 2019, 7, 3842.

[10] Yang, W. J.; Luo, N. J.; Zheng, C.; Huang, S. P.; Wei, M. D. Small 2019, 15, 1903904. 